\title{
Comparison of minimally invasive direct coronary artery bypass and drug-eluting stents for management of isolated left anterior descending artery disease: a systematic review and meta-analysis of 7,710 patients
}

\author{
Shahzad G. Raja ${ }^{1}$, Mohsin Uzzaman ${ }^{2}$, Sheena Garg ${ }^{1}$, Gowthanan Santhirakumaran ${ }^{1}$, Michelle Lee ${ }^{3}$, \\ Manish K. Soni ${ }^{1}$, Habib Khan ${ }^{4}$ \\ ${ }^{1}$ Department of Cardiac Surgery, Harefield Hospital, London, UK; ${ }^{2}$ Department of Cardiac Surgery, Queen Elizabeth Hospital, Birmingham, UK; \\ ${ }^{3}$ Department of Gastroenterology, Hillingdon Hospital, London, UK; ${ }^{4}$ Department of Cardiac Surgery, St. George's Hospital, London, UK \\ Correspondence to: Shahzad G. Raja. Department of Cardiac Surgery, Harefield Hospital, Hill End Rd, Harefield, Uxbridge UB9 6JH, London, UK. \\ Email: drrajashahzad@hotmail.com.
}

Background: In the contemporary era of drug-eluting stents (DES) the issue of optimal revascularization strategy for patients with isolated disease of the left anterior descending (LAD) artery has gained more significance. We aimed to evaluate the current best evidence on the subject by performing a systematic review and meta-analysis of the studies comparing minimally invasive direct coronary artery bypass (MIDCAB) grafting with DES in patients with isolated LAD disease.

Methods: A literature search was conducted from 1966 through March 2018 using MEDLINE, EMBASE, and other scientific databases to identify relevant articles. Analyzed outcomes included all-cause mortality, myocardial infarction (MI), major adverse cardiac and cerebrovascular events (MACCE) and target vessel revascularization (TVR). The random effects model was used to calculate the outcomes of both binary and continuous data to control any heterogeneity between the studies. Heterogeneity amongst the trials was determined by means of the Cochran $\mathrm{Q}$ value and quantified using the $\mathrm{I}^{2}$ inconsistency test. All $\mathrm{P}$ values were 2-sided and a $5 \%$ level was considered significant.

Results: A total of 7,710 patients from 12 studies were identified. There was no significant difference in mortality rate [pooled odds ratio $(\mathrm{OR})=0.92 ; 95 \%$ confidence interval $(\mathrm{CI}), 0.65$ to $1.32 ; \mathrm{P}=0.66$ ], MI rate (pooled OR $=1.13,95 \% \mathrm{CI}, 0.62$ to 2.06 ; $\mathrm{P}=0.69$ ) or MACCE (pooled OR $=1.31$; $95 \% \mathrm{CI}, 0.58$ to 2.95 ; $\mathrm{P}=0.52$ ). However, TVR rate was significantly more for patients having percutaneous intervention with DES (pooled OR $=0.27 ; 95 \% \mathrm{CI}, 0.16$ to $0.45 ; \mathrm{P}<0.0001$ ) compared to MIDCAB.

Conclusions: MIDCAB offers superior freedom from TVR with similar mortality, MI rate, and MACCE compared to percutaneous intervention with DES for revascularization in patient with isolated proximal LAD stenosis.

Keywords: Proximal left anterior descending artery; minimally invasive direct coronary artery bypass grafting (MIDCAB grafting); minimally invasive direct coronary artery bypass (MIDCAB); minimally invasive revascularization; left internal mammary artery

Submitted Apr 13, 2018. Accepted for publication Jun 18, 2018.

doi: $10.21037 /$ acs.2018.06.16

View this article at: http://dx.doi.org/10.21037/acs.2018.06.16 


\section{Introduction}

Percutaneous coronary intervention with stents and minimally invasive direct coronary artery bypass (MIDCAB) grafting are well-established strategies for patients with isolated proximal stenosis of left anterior descending (LAD) artery (1). However, several studies published in literature have consistently proven that the incidence of mediumterm adverse events and repeat revascularization of the target lesion were higher after percutaneous intervention with bare metal stents than MIDCAB in these patients (2-5). The introduction of drug-eluting stents (DES) in clinical practice has shown a great deal of promise for the treatment of both de novo and restenotic lesions, with reduction in instent neointimal proliferation that causes restenosis, thereby reducing the incidence of symptomatic recurrence to less than $5 \%$, rivaling that of bypass surgery (6).

Currently, the evidence comparing DES with MIDCAB is limited to only a small number of studies with small sample sizes, divergent designs and conflicting results. Each one of these studies provides a piece of evidence that, when taken together with others, addresses important questions for patients, clinicians, and other healthcare decision-makers. In the current era of evidence-based medicine, pairwise meta-analyses are increasingly used to synthesize the results of different trials evaluating the same intervention(s) to obtain an overall estimate of the treatment effect of one intervention relative to the control (7). We aimed to evaluate the current best evidence by performing a systematic review and meta-analysis of the studies comparing the efficacy of MIDCAB with DES in patients with isolated LAD disease.

\section{Methods}

\section{Literature search strategy}

MEDLINE, EMBASE, Cochrane Controlled Trials Register (CCTR), Cochrane Database of Systematic Reviews (CDSR), Database of Abstracts of Reviews of Effects (DARE), Science Citation Index (SCI), Current Contents, NHS Economic Evaluation Database (NEED), and International Network of Agencies for Health Technology Assessment (INAHTA) databases were searched from the date of their inception to March 10, 2018, using the search terms: (drug eluting stent or DES or percutaneous coronary intervention or PCI) AND (minimally invasive direct coronary artery bypass grafting or MIDCAB or left internal mammary artery or LIMA)
AND (left anterior descending or LAD). The search was undertaken in accordance with Cochrane Collaboration recommendations (8) and was aimed at finding all published reports comparing MIDCAB with percutaneous coronary intervention using DES for revascularization of patients with isolated LAD stenosis. Articles written in English only were included. Tangential electronic exploration of related articles and hand searches of bibliographies, scientific meeting abstracts, and related journals were also performed.

\section{Eligibility criteria}

Studies written in English only comparing MIDCAB with percutaneous coronary intervention using DES recruiting participants with isolated proximal LAD disease evaluating one or more of the following outcomes: mortality, myocardial infarction (MI), target vessel revascularization (TVR) or a composite outcome namely major adverse cardiac and cerebrovascular events (MACCE) were included.

Studies recruiting participants with multivessel disease, studies where PCI was undertaken using bare metal stents, and reviews with no original data were excluded.

\section{Data extraction and critical appraisal}

The search results were reviewed by two independent judicators (SG, GS) for studies that met the inclusion criteria. The studies identified were validated by SGR. The studies fulfilling the inclusion criteria found by the search strategy were then evaluated. Each paper was subjected to a structured analysis using critical appraisal checklists (9). Such checklists are widely available in several formats and are helpful in assessing the methodological and analytical soundness of a trial and in uncovering any serious methodological flaws (9). The information extracted from each study included the study design, country, year of study, number of participants, age of participants, percentage of male participants, participant selection criteria, stent type, operation, outcomes, follow-up, and key outcomes.

\section{Statistical analysis}

Data from the individual eligible studies were entered into a spreadsheet for further analysis. Weighted mean differences (WMD) were calculated for the effect size of continuous variables. Pooled odds ratios (OR) were calculated for discrete variables such as mortality rates. The random- 


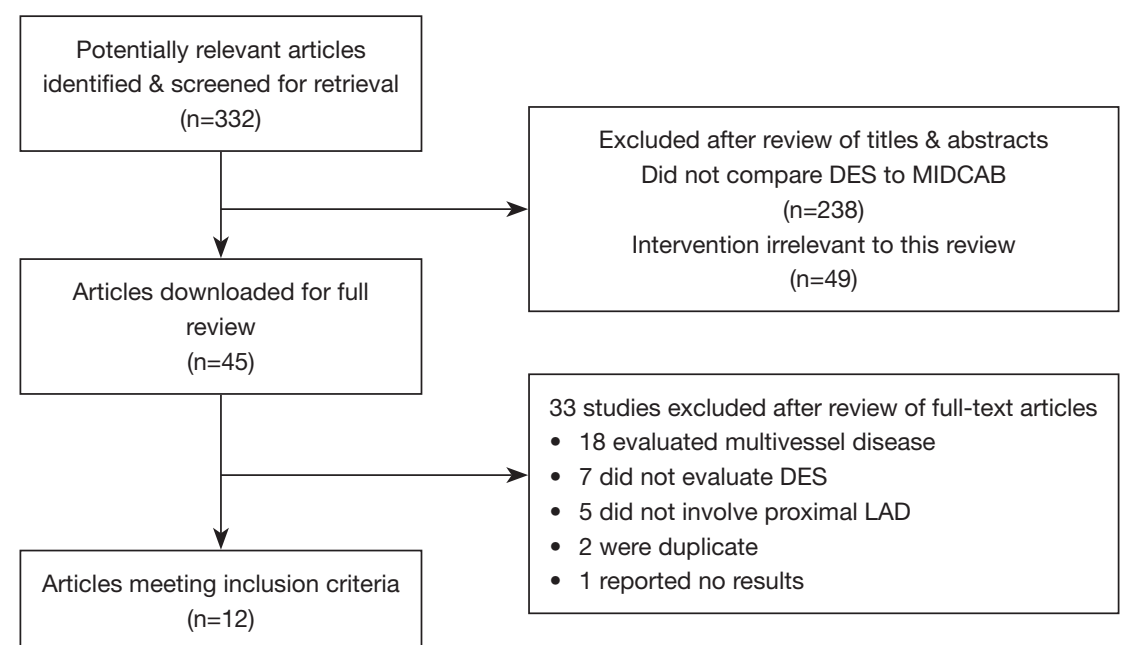

Figure 1 Flow chart depicting study selection for meta-analysis. DES, drug-eluting stents; LAD, left anterior descending; MIDCAB, minimally invasive direct coronary artery bypass.

effects models (DerSimonian Laird) were used to calculate the outcomes of both binary and continuous data to control any heterogeneity between the studies. Heterogeneity amongst the trials was determined by means of the Cochran $\mathrm{Q}$ value and quantified using the $\mathrm{I}^{2}$ inconsistency test. In this study, we did not perform meta-regression or sensitivity analysis because of the small number of studies included. All $\mathrm{P}$ values were 2 -sided and a 5\% level was considered significant. StatsDirect 2.5.7 (StatsDirect, Altrincham, UK) was used to perform the statistical analysis.

\section{Results}

\section{Quantity of evidence}

Our search yielded 332 relevant reports, and after screening and reviewing full manuscripts, 12 studies met the inclusion criteria (10-21). The process of study selection is shown in Figure 1. There were a total of 7,710 patients with 2,553 in the MIDCAB group compared to 5,157 in the DES group.

\section{Quality of evidence}

The study designs and participant characteristics of the included studies are listed in Tables 1,2. There were three randomized controlled trials (RCTs) $(11,18,21)$ and nine cohort studies $(10,12-17,19,20)$. Most studies had $>100$ participants in each arm except for four studies $(11,14,18,21)$. Six retrospective studies reported reliable methods for ascertaining outcomes (10-14,20), whereas the remaining studies did not report how outcomes were ascertained. All three RCTs $(11,18,21)$ had similar baseline characteristics among participants.

\section{Assessment of outcomes}

\section{Study outcomes are listed in Table 3 Mortality}

Ten studies reported on the mortality rate. There were 2,334 patients in the MIDCAB group and 5,042 patients in the DES group. There was no statistical heterogeneity between studies (Cochran Q $=16.0, \mathrm{P}=0.07 ; \mathrm{I}^{2}=43.8 \%$, 95\% CI, $0 \%$ to $71.5 \%$ ). In the random effects model, there was no significant difference in the mortality rates between the MIDCAB and DES groups [pooled OR $=0.92 ; 95 \%$ confidence interval (CI), 0.65 to $1.32 ; \mathrm{P}=0.66]$ (Figure 2).

\section{MI}

Six studies assessed the rate of MI. There were 761 patients in the MIDCAB group and 749 patients in the DES group. There was no statistical heterogeneity between studies (Cochran $\mathrm{Q}=4.92, \mathrm{P}=0.43 ; \mathrm{I}^{2}=0 \%, 95 \% \mathrm{CI}, 0 \%$ to $61 \%$ ). In the random effects model, there was no significant differences in the rates of MI in the MIDCAB group compared to DES group (pooled OR $=1.13,95 \% \mathrm{CI}, 0.62$ to 2.06; $\mathrm{P}=0.69$ ) (Figure 3).

\section{MACCE}

Six studies reported on the rate of MACCE for the two groups. There were 1,353 patients in the MIDCAB group and 1,922 in the DES group. There was significant 
Table 1 Study design, participant characteristics and revascularization strategies

\begin{tabular}{|c|c|c|c|c|c|c|}
\hline First author [year] & Country & Study design & Study period & $\begin{array}{l}\text { Total participants } \\
\text { (\% male) }\end{array}$ & Mean age & $\begin{array}{l}\text { Revascularization } \\
\text { strategies }\end{array}$ \\
\hline lqbal [2017] & UK & Retrospective & 2004-2015 & 3,473 (79.1\%) & 63 & $\begin{array}{l}\text { MIDCAB vs. FDES } \\
\text { MIDCAB vs. SDES }\end{array}$ \\
\hline Blazek [2015] & Germany & RCT & 2003-2014 & 129 (70\%) & 66 & MIDCAB vs. FDES ${ }^{c}$ \\
\hline Hannan [2014] & USA & Retrospective & 2008-2011 & $1,430(66 \%)$ & NR & MIDCAB vs. DES* \\
\hline Ungureanu [2013] & Belgium & Retrospective & NR & 204 (NR) & NR & MIDCAB vs. SDES $^{d}$ \\
\hline Jones [2011] & UK & Retrospective & 2003-2010 & 874 (NR) & NR & MIDCAB vs. DES* \\
\hline Buszman [2011] & Poland & Retrospective & 2004-2009 & $463(75 \%)$ & 61 & $\begin{array}{l}\text { MIDCAB vs. FDES }{ }^{a} \\
\text { MIDCAB vs. SDES }^{b}\end{array}$ \\
\hline Glineur [2009] & Belgium & Retrospective & NR & 350 (NR) & 63 & MIDCAB vs. DES* \\
\hline Toutouzas [2007] & Greece & Retrospective & 2001-2006 & $257(86 \%)$ & 61 & $\begin{array}{l}\text { MIDCAB vs. FDES } \\
\text { MIDCAB vs. SDES }\end{array}$ \\
\hline Hong [2005] & South Korea & RCT & 2003 & $189(64 \%)$ & 61 & MIDCAB vs. FDES $^{a}$ \\
\hline
\end{tabular}

Table 2 Technical aspects of surgical revascularization of LAD for the included studies

\begin{tabular}{|c|c|c|c|}
\hline Study & Use of CPB & Use of LIMA & Conversion to full sternotomy \\
\hline lqbal et al. (10) & NR & $96 \%^{a}$ & NR \\
\hline Blazek et al. (11) & No & $100 \%$ & No \\
\hline Hannan et al. (12) & NR & NR & NR \\
\hline Benedetto et al. (13) & No & $100 \%$ & No \\
\hline Jones et al. (15) & No & $100 \%$ & No \\
\hline Buszman et al. (16) & No & $100 \%$ & No \\
\hline Patsa et al. (17) & No & $100 \%$ & No \\
\hline Thiele et al. (18) & No & $98.5 \%{ }^{b}$ & Yes $(6.2 \%)$ \\
\hline Hong et al. (21) & No & $100 \%$ & No \\
\hline
\end{tabular}


Table 3 Duration of follow-up and key outcomes of included studies

\begin{tabular}{|c|c|c|}
\hline Study & Follow-up & Key results (MIDCAB vs. DES) \\
\hline \multirow[t]{2}{*}{ lqbal [2017] } & 3 years & Similar mortality $17 / 378$ vs. $82 / 275$ (FDES; HR $1.16,95 \% \mathrm{Cl}, 0.67$ to $2.02, \mathrm{P}=0.597$ ) \\
\hline & & $17 / 378$ vs. $68 / 1,061$ (SDES; HR 0.98, $95 \% \mathrm{Cl}, 0.53$ to $1.82, \mathrm{P}=0.946$ ) \\
\hline \multirow{2}{*}{ Blazek [2015] } & & Death 11 vs. 9, RR 0.91 (0.58-1.39) \\
\hline & & MI 6 vs. 4, RR 0.83 (0.48-1.41) \\
\hline \multirow[t]{3}{*}{ Hannan [2014] } & 3 years & Composite 56/715 vs. 46/715, aHR 1.15 (0.76-1.73) \\
\hline & & Death 39 vs. 34, aHR $1.14(0.70-1.85)$ \\
\hline & & TVR 42 vs. 77, aHR $0.54(0.36-0.81)$ \\
\hline Benedetto [2014] & 2,232 days & Death $13 / 303$ vs. 31/303, HR 2.19 (1.15-4.17) \\
\hline \multirow[t]{3}{*}{ Jones [2011] } & 4 years & MACE $15 / 122$ vs. $89 / 752$ \\
\hline & & Death 10 vs. 32 \\
\hline & & TVR 3 vs. 44 \\
\hline \multirow[t]{3}{*}{ Buszman [2011] } & 5 years & $\begin{array}{l}\text { MACCE } 71 / 276 \text { vs. 62/178 } \\
\text { Death } 12 \text { vs. } 12\end{array}$ \\
\hline & & MI 12 vs. 9 \\
\hline & & TVR 38 vs. 67 \\
\hline Patsa [2010] & 26 months & Death $2 / 110$ vs. $5 / 302$ \\
\hline \multirow{3}{*}{ Glineur [2009] } & & Death 7 vs. 7 , HR $0.76(0.25-2.30)$ \\
\hline & & MI 2 vs. 5, HR 0.25 (0.03-2.36) \\
\hline & & TVR 5 vs. 26, HR 0.19 (0.07-0.49) \\
\hline \multirow[t]{4}{*}{ Toutouzas [2007] } & 18 months & MACE $3 / 110$ vs. $4 / 147$ \\
\hline & & Death 2 vs. 3 \\
\hline & & MI 1 vs. 0 \\
\hline & & TVR 0 vs. 3 \\
\hline \multirow[t]{3}{*}{ Hong [2005] } & 6 months & Death 2/68 vs. 0/116 \\
\hline & & MI 2 vs. 2, RR 0.73 (0.27-1.99) \\
\hline & & TVR 1 vs. 3, RR 1.49 (0.27-8.22) \\
\hline
\end{tabular}

$\mathrm{CI}$, confidence interval; DES, drug eluting stent; FDES, first generation drug-eluting stent; HR, hazard ratio; LIMA, left internal mammary artery; MACE, major adverse cardiovascular events; MACCE, major adverse cardiovascular or cerebrovascular event; MI, myocardial infarction; MIDCAB, minimally invasive direct coronary artery bypass; RR, relative risk; SDES, second generation drug-eluting stent; TVR, target vessel revascularization. 
Odds ratio meta-analysis plot [random effects]

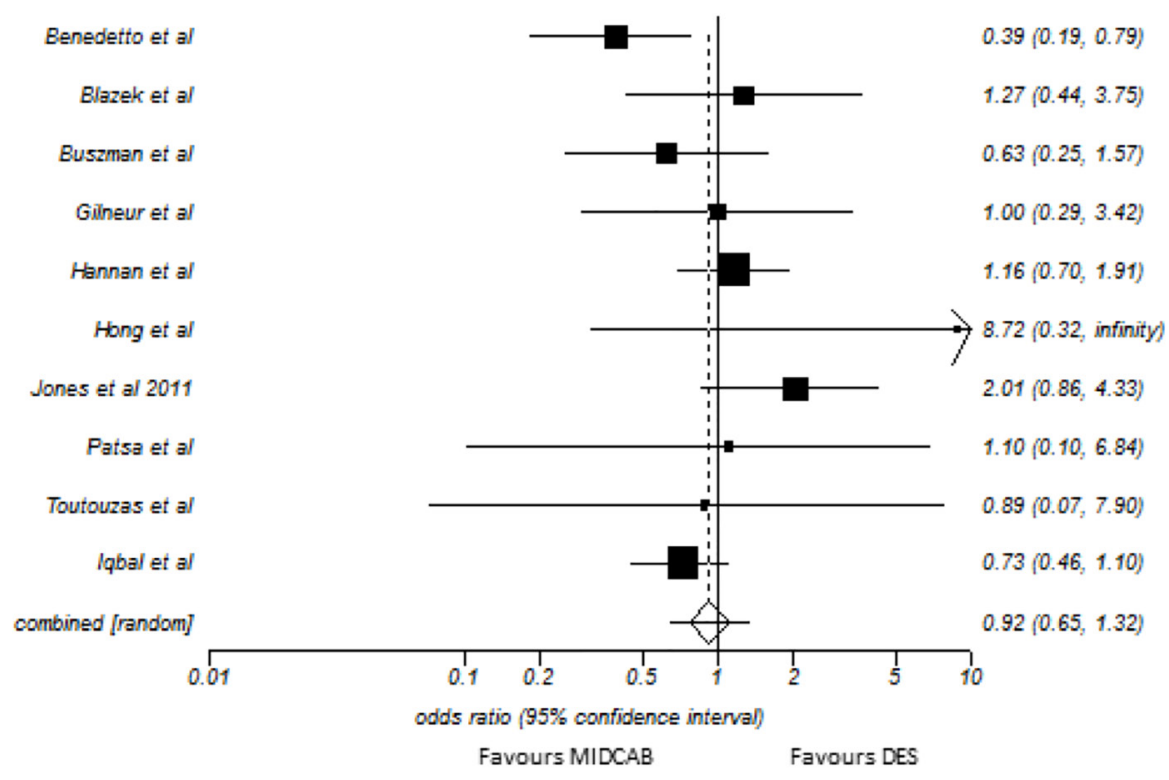

Figure 2 Risk of mortality with MIDCAB versus DES for proximal LAD disease. DES, drug-eluting stents; LAD, left anterior descending; MIDCAB, minimally invasive direct coronary artery bypass.

Odds ratio meta-analysis plot [random effects]

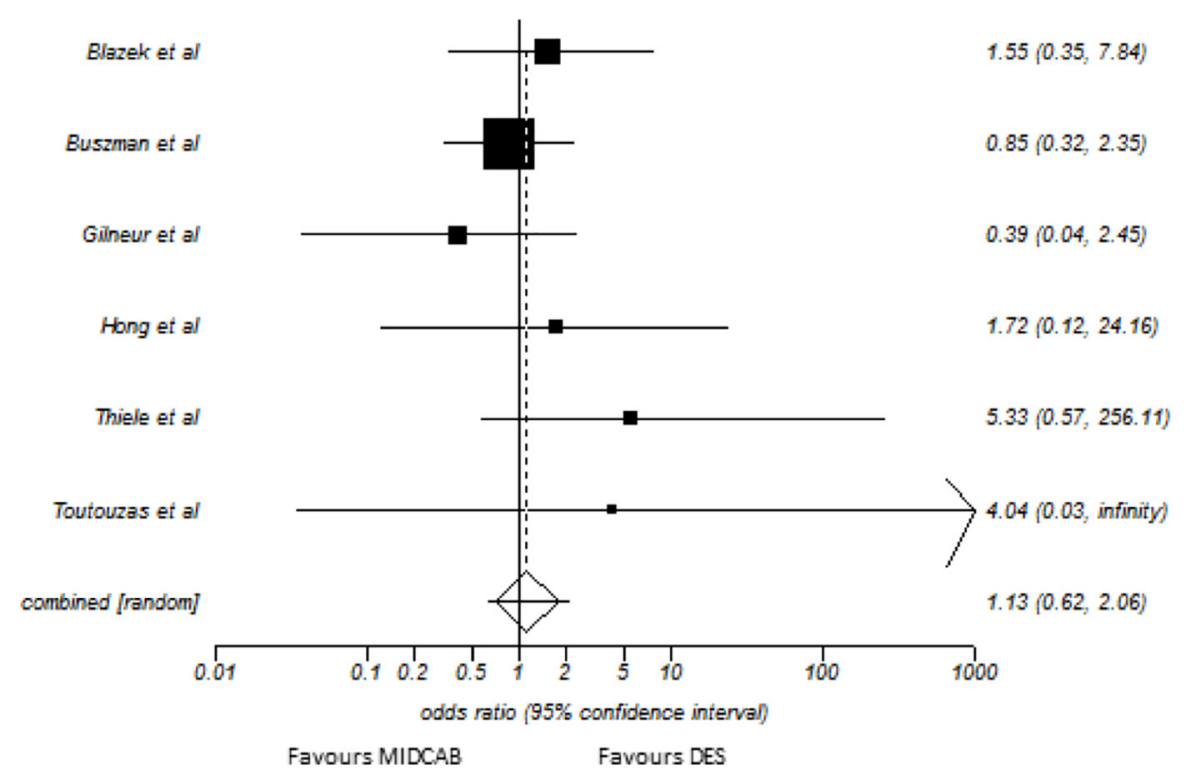

Figure 3 Risk of myocardial infarction with MIDCAB versus DES for proximal LAD disease. DES, drug-eluting stents; LAD, left anterior descending; MIDCAB, minimally invasive direct coronary artery bypass. 
statistical heterogeneity between the six studies (Cochran $\mathrm{Q}=38.8, \mathrm{P}=0<0.0001 ; \mathrm{I}^{2}=87.1 \%, 95 \% \mathrm{CI}, 72.4 \%$ to 92.3\%) (Figure 4). In the random effect model, there was no significant difference in the MACCE rates between the

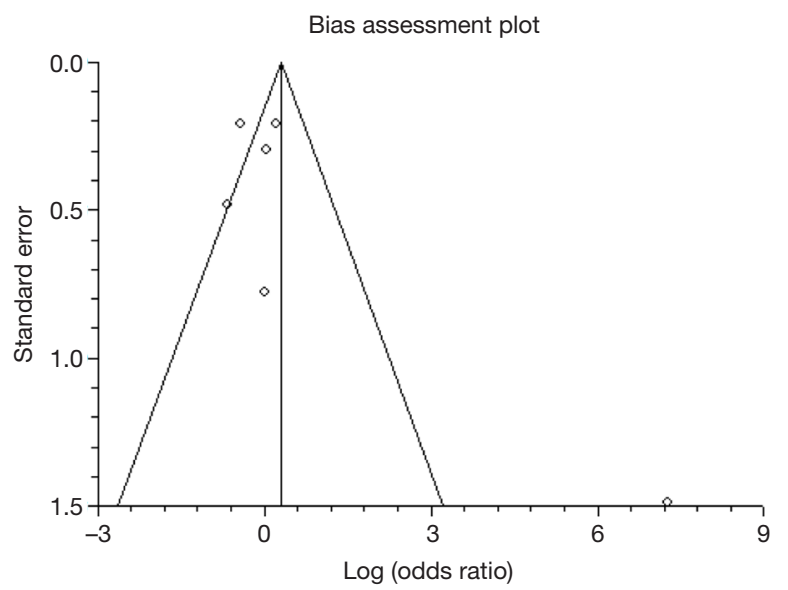

Figure 4 Funnel plot demonstrating heterogeneity amongst studies reporting MACCE rates with MIDCAB versus DES for proximal LAD disease. DES, drug-eluting stents; LAD, left anterior descending; MIDCAB, minimally invasive direct coronary artery bypass.
CABG group and PCI groups (pooled OR $=1.31 ; 95 \% \mathrm{CI}$, 0.58 to $2.95 ; \mathrm{P}=0.52)$ (Figure 5).

\section{TVR}

Eight studies reported on the rate of TVR between the two groups. There were 999 patients in the CABG group and 1,803 patients in the PCI groups. There was no significant statistical heterogeneity between the eight studies (Cochran $\mathrm{Q}=3.47, \mathrm{P}=0.84 ; \mathrm{I}^{2}=0 \%$; $95 \% \mathrm{CI}, 0 \%$ to $56.3 \%)$. In the random effect model, there was significant difference in the TVR rates between the CABG groups and PCI groups (pooled OR $=0.27$; $95 \%$ CI, 0.16 to 0.45 ; $\mathrm{P}<0.0001$ ) (Figure 6).

\section{Discussion}

This systematic review and meta-analysis of three RCTs and nine cohort studies enrolling 7,710 participants is the most up to date meta-analysis on the subject. The results of the analysis suggest both MIDCAB and DES are effective strategies for revascularization of isolated LAD stenosis. However, DES compared to MIDCAB is associated with increased TVR.

The results of this meta-analysis substantiate those of a previously published meta-analysis by Kinnaird et al. (22).

\section{Odds ratio meta-analysis plot [random effects]}

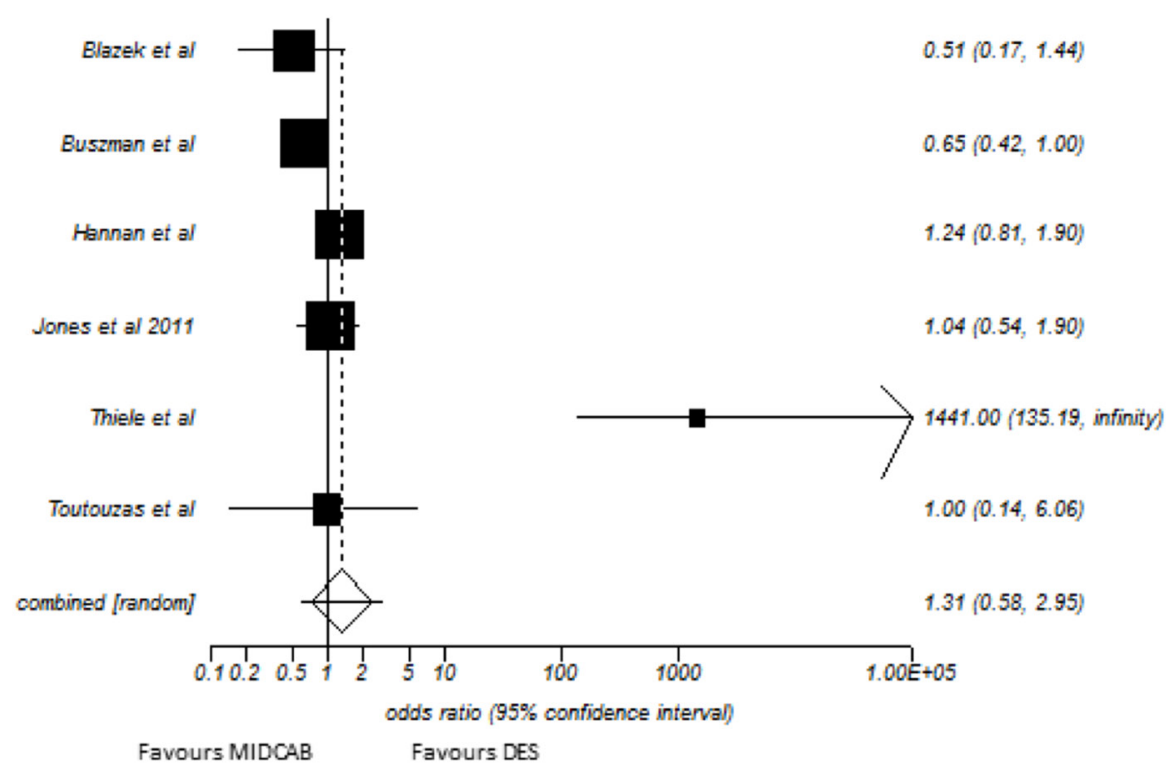

Figure 5 Risk of MACCE with MIDCAB versus DES for proximal LAD disease. DES, drug-eluting stents; LAD, left anterior descending; MIDCAB, minimally invasive direct coronary artery bypass; MACCE, major adverse cardiac and cerebrovascular events. 
Odds ratio meta-analysis plot [random effects]

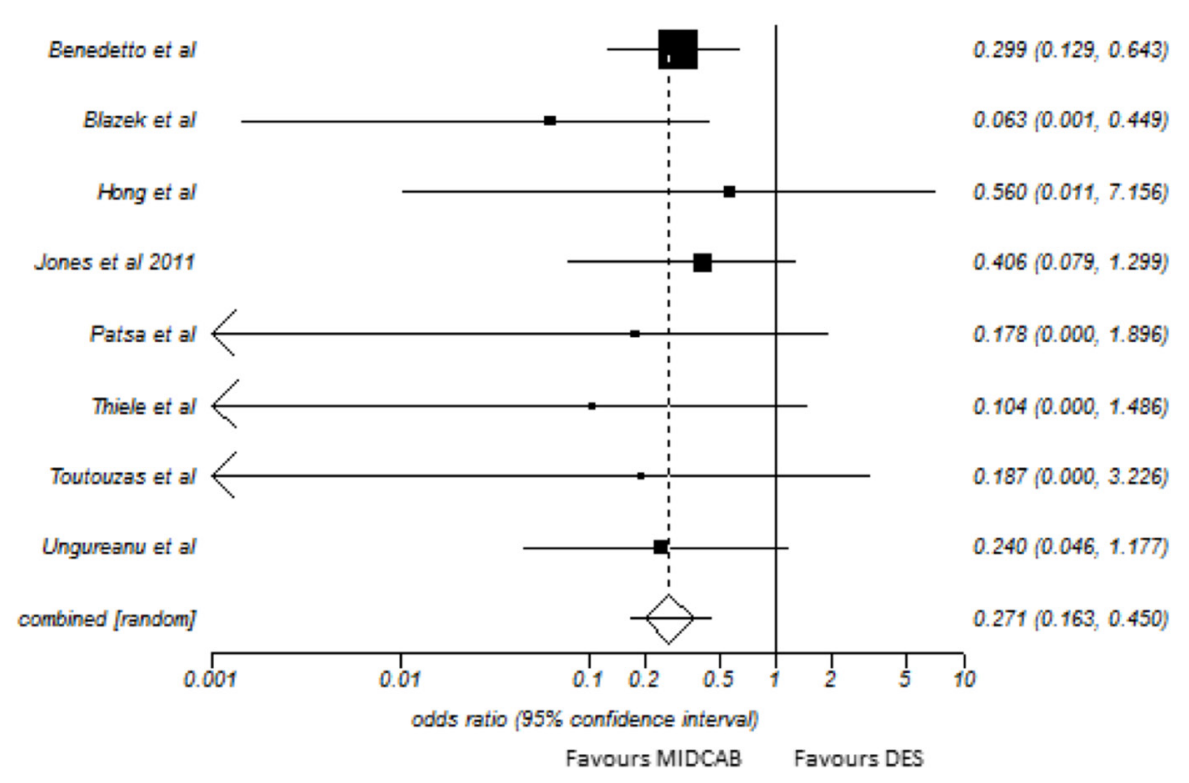

Figure 6 Risk of TVR with MIDCAB versus DES for proximal LAD disease. DES, drug-eluting stents; LAD, left anterior descending; MIDCAB, minimally invasive direct coronary artery bypass; TVR, target vessel revascularization.

This only other meta-analysis comparing MIDCAB with DES reported no significant difference in mortality between the two interventions [MIDCAB: 111 of 2,122 (5.2\%) and DES: 120 of 2,574 (4.7\%); relative risk (RR) 1.23 ; $95 \% \mathrm{CI}$, 0.90 to 1.69$]$. For the composite outcome MACE, DES was associated with significant increase in adverse events (RR 1.41 ; $95 \%$ CI, 1.03 to $1.93,8$ studies, 4,230 participants). There was no significant difference in the risk of MI (RR 0.86 ; $95 \%$ CI, 0.58 to 1.26 ) between the 2 groups. There were 239 TVR events among 2,237 participants in the DES group (10.7\%) and 145 TVR events among 2,793 participants in the MIDCAB group (5.2\%) with a significant increased risk of TVR in the DES group (RR 2.52; 95\% CI, 1.69 to $3.77,5,030$ participants) compared with MIDCAB.

The interpretation of this meta-analysis will need to take into consideration the fact that the DES technology used in several of the included studies is no longer representative of the contemporary percutaneous coronary intervention practice. However, this meta-analysis confirms the superiority of LIMA-to-LAD in terms of freedom from repeat re-intervention against both first- and secondgeneration DES making it an impressive benchmark against which all existing and future DES technologies must perform. Despite this, it will not be inappropriate to suggest that each case should be treated on their individual merits, and the information about significant excess of repeat TVR with DES strategy must feature in the informed consent.

There are several important caveats that must be acknowledged. First, as studies with different designs were included therefore some results of our meta-analysis have significant heterogeneities. Second, definitions of end points were different across included studies. Third, follow-up lengths were variable with majority of the studies reporting follow-up up to 1 year. Hence, the long-term durability of DES particularly second-generation DES versus MIDCAB remains undetermined. Fourth, it is important to point out the publication bias exaggerating the positive effects of MIDCAB could be a likely explanation for the superiority of MIDCAB over DES in terms of TVR as positive results have tendency to be published more likely than negative results. Fifth, data about the timing of intervention was not collected. The possibility of DES being offered preferentially to patients presenting with acute coronary syndrome and MIDCAB to elective patients in retrospective studies could also account for some of the perceived advantages of MIDCAB. Last and not the least, one could argue that the differences in TVR between DES and MIDCAB could be because of the difference 
in extent and complexity of coronary artery disease. The purists could argue that larger RCTs with longer followup are required to improve the evidence-base. However, at the same time, it is extremely important to mention that RCTs of impractical sizes with extremely long followup will be required to conclusively prove the superiority of any myocardial revascularization strategy or technique. Furthermore, such studies are unlikely to be performed because the rate of technological advancement particularly in the field of percutaneous coronary intervention is likely to make the results of such a trial obsolete.

This most up-to-date meta-analysis suggests that MIDCAB offers superior freedom from TVR with similar mortality, MI rate and MACCE compared to percutaneous intervention with DES for revascularization in patient with isolated proximal LAD stenosis.

\section{Acknowledgements}

None.

\section{Footnote}

Conflicts of Interest: The authors have no conflicts of interest to declare.

\section{References}

1. Goy JJ, Eeckhout E, Burnand B, et al. Coronary angioplasty versus left internal mammary artery grafting for isolated proximal left anterior descending artery stenosis. Lancet 1994;343:1449-53.

2. Goy JJ, Kaufmann U, Hurni M, et al. 10-year followup of a prospective randomized trial comparing baremetal stenting with internal mammary artery grafting for proximal, isolated de novo left anterior coronary artery stenosis the SIMA (Stenting versus Internal Mammary Artery grafting) trial. J Am Coll Cardiol 2008;52:815-7.

3. Drenth DJ, Winter JB, Veeger NJ, et al. Minimally invasive coronary artery bypass grafting versus percutaneous transluminal coronary angioplasty with stenting in isolated high-grade stenosis of the proximal left anterior descending coronary artery: six months' angiographic and clinical follow-up of a prospective randomized study. J Thorac Cardiovasc Surg 2002;124:130-5.

4. Shirai K, Lansky AJ, Mehran R, et al. Minimally invasive coronary artery bypass grafting versus stenting for patients with proximal left anterior descending coronary artery disease. Am J Cardiol 2004;93:959-62.

5. Thiele H, Oettel S, Jacobs S, et al. Comparison of baremetal stenting with minimally invasive bypass surgery for stenosis of the left anterior descending coronary artery: a 5-year follow-up. Circulation 2005;112:3445-50.

6. Raja SG. Drug-eluting stents and the future of coronary artery bypass surgery: facts and fiction. Ann Thorac Surg 2006;81:1162-71.

7. IntHout J, Ioannidis JP, Borm GF. Obtaining evidence by a single well-powered trial or several modestly powered trials. Stat Methods Med Res 2016;25:538-52.

8. Higgins JP, Green S. Cochrane Handbook for Systematic Reviews of Interventions Version 5.1.0 [updated March 2011]. The Cochrane Collaboration, 2011. Available online: http://handbook.cochrane.org

9. BestBETS critical appraisal checklists. Available online: http://www.bestbets.org/links/BET-CA-worksheets.php

10. Iqbal MB, Ilsley C, De Robertis F, et al. Comparison of outcomes of coronary artery bypass grafting using internal mammary graft versus percutaneous coronary intervention for isolated proximal left anterior descending narrowing. Am J Cardiol 2017;119:719-26.

11. Blazek S, Rossbach C, Borger MA, et al. Comparison of sirolimus-eluting stenting with minimally invasive bypass surgery for stenosis of the left anterior descending coronary artery: 7-year follow-up of a randomized trial. JACC Cardiovasc Interv 2015;8:30-8.

12. Hannan EL, Zhong Y, Walford G, et al. Coronary artery bypass graft surgery versus drug-eluting stents for patients with isolated proximal left anterior descending disease. J Am Coll Cardiol 2014;64:2717-26.

13. Benedetto U, Raja SG, Soliman RF, et al. Minimally invasive direct coronary artery bypass improves late survival compared with drug-eluting stents in isolated proximal left anterior descending artery disease: a 10year follow-up, single-center, propensity score analysis. J Thorac Cardiovasc Surg 2014;148:1316-22.

14. Ungureanu C, Laruelle C, Khoury E, et al. Retrospective comparison of minimally invasive direct coronary artery bypass surgery versus Xience drug eluting stents in isolated proximal left anterior descending coronary artery stenosis. Acta Cardiol 2013;68:104-5.

15. Jones D, Rathod K, Gallagher S, et al. Drug eluting stents in the treatment of isolated proximal LAD disease are associated with similar outcomes compared to minimally invasive LIMA grafts. J Am Coll Cardiol 2011;58:B56.

16. Buszman P, Król L, Cisowski M, et al. DES vs. MIDCAB 
for proximal LAD disease: long term registry results. J Am Coll Cardiol 2011;58:20:B53.

17. Patsa C, Toutouzas K, Tsiamis E, et al. Long term clinical outcome after DES implantation or LIMA grafting in patients with an isolated pLAD lesion. Eur Heart J 2010;31:19.

18. Thiele H, Neumann-Schniedewind P, Jacobs S, et al. Randomized comparison of minimally invasive direct coronary artery bypass surgery versus sirolimuseluting stenting in isolated proximal left anterior descending coronary artery stenosis. J Am Coll Cardiol 2009;53:2324-31.

19. Glineur D, Boodhwani M, Noirhomme P, et al. Short and midterm clinical outcome following single vessel LAD revascularization with MIDCAB versus DES. ISMICS 2009;3:159.

Cite this article as: Raja SG, Uzzaman M, Garg S, Santhirakumaran G, Lee M, Soni MK, Khan H. Comparison of minimally invasive direct coronary artery bypass and drug-eluting stents for management of isolated left anterior descending artery disease: a systematic review and meta-analysis of 7,710 patients. Ann Cardiothorac Surg 2018;7(5):567-576. doi: 10.21037/acs.2018.06.16
20. Toutouzas K, Patsa C, Vaina S, et al. Drug eluting stents versus coronary artery bypass surgery in patients with isolated proximal lesion in left anterior descending artery suffering from chronic stable angina. Catheter Cardiovasc Interv 2007;70:832-7.

21. Hong SJ, Lim DS, Seo HS, et al. Percutaneous coronary intervention with drug-eluting stent implantation vs. minimally invasive direct coronary artery bypass (MIDCAB) in patients with left anterior descending coronary artery stenosis. Catheter Cardiovasc Interv 2005;64:75-81.

22. Kinnaird T, Kwok CS, Narain A, et al. Meta-Analysis of Percutaneous coronary intervention with drug-eluting stent versus coronary artery bypass grafting for isolated proximal left anterior descending coronary disease. Am J Cardiol 2016;118:1171-7. 